\title{
EFFECTS OF CELL PHONE CONVERSATION DIFFICULTY ON DRIVING PERFORMANCE
}

\author{
Mick Rakauskas \\ HumanFIRST Program, ITS Institute \\ University of Minnesota \\ 1103 Mech. Eng., 111 Church Street SE \\ Minneapolis, MN 55455 USA \\ E-mail: mickr@me.umn.edu \\ Dr. Leo Gugerty \\ Clemson University
}

\begin{abstract}
The literature has shown that conversations and verbal tasks degrade the driver's ability to maintain control of the vehicle and avoid hazardous conditions. However, the question of how the difficulty (or intensity) of a conversation relates to decrements in driving performance needs further investigation. Other studies have shown that conversations may hinder driving, but these were unable to quantify a difficulty threshold at which conversations and verbal tasks became more hazardous. This study compared two quantifiably different levels of conversation difficulty and a non-conversing condition over measures of driving performance and mental workload.

This study used a GlobalSim Corporation driving simulator, allowing participants full control of the vehicle on two lane roads in a rural setting. Driving conditions were set up and controlled in order to determine the extent to which conversation had an effect on driving performance, which was assessed in terms of steering and speed-maintenance ability and the ability to deal with hazardous situations. We compared driving performance when participants were not conversing to when they were conversing, as well as whether a more difficult conversation had a greater effect on performance than an easier one. Participants conversed with the experimenter over a hands-free headset. Conversations consisted of answering and conversing based on either easy ("small talk") or difficult ("thought provoking") questions.
\end{abstract}

Participants drove a simulated car for approximately thirty minutes, with ten minutes devoted to driving under each of the conversation conditions (no talking, easy conversation, difficult conversation). During each of the ten minute driving sessions, participants were exposed to one of three hazardous events: an ambulance running a red light in front of the driver, an oncoming car swerving into the driver's lane, and a parallel-parked car pulling out in front of the driver. A variety of variables were measured in the categories of speed maintenance (accelerator position variability, speed variability, average speed), lane position maintenance (steering offset, average lateral speed), crash avoidance (collisions, response time to hazardous events), and mental workload (RSME). Two double multivariate ANOVAs were conducted, and then planned contrast analyses were used to test how the conversation levels affected each dependent measure.

While concurrently driving and conversing, participants had higher variation in their steering and speed than when driving without conversing. While driving and conversing, participants also drove at slower average speeds and reported having to exert higher mental effort. No significant 
differences between conversation and non-conversation conditions were found for collisions or response time to hazards. When comparing the difficult and easy conversation conditions, the only significant difference in driving performance was for speed variation — participants showed more speed variation during difficult than during easy conversations.

The findings from this study suggest that having a conversation over a hands-free phone while driving may cause decrements in steering and speed maintenance performance. Also, people thought that talking on a cell phone while driving was more mentally demanding than driving while not talking. These findings suggest that regardless of conversation intensity, driving performance will be affected by this attentional distraction both through actual decrements in performance as well as in perceived distraction from the driving task. It seems to be something about the act of talking, as opposed to the content of the material, that is detrimental to driving performance. This is consistent with other research (Briem \& Hedman, 1995; Irwin, Fitzgerald, \& Burg, 2000; McKnight \& McKnight, 1993) that has found effects for conversations but little or no effect of varying conversational difficulty.

\section{REFERENCES}

Briem, V., Hedman, L. (1995). Behavioural effects of mobile telephone use during simulated driving. Ergonomics, 38(12), 2536-2562.

Irwin, M., Fitzgerald, C., \& Berg, W. P. (2000). Effect of the intensity of wireless telephone conversations on reaction time in a braking response. Perceptual and Motor Skills, 2000 (90), $1130-1134$.

McKnight, A. J., McKnight, A. S. (1993). The effect of cellular phone use upon driver attention. Accident Analysis and Prevention, 25 (3), 259-265. 